\title{
Mechanism of allostery and catalysis in P. falciparum GMP Synthetase
}

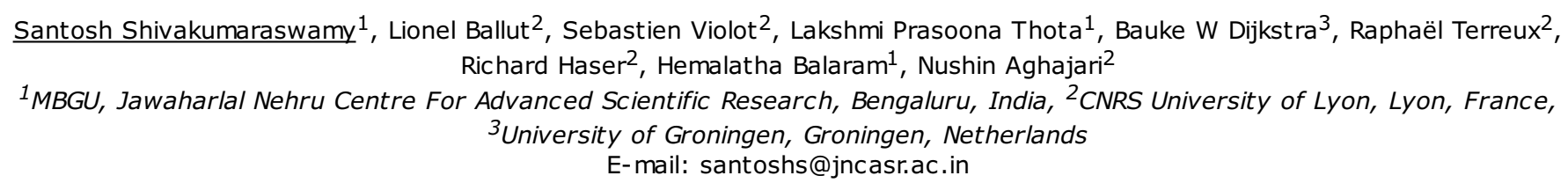

More than 200 million cases of malaria were reported worldwide in the year 2015. The members of the genus Plasmodia are the causative agents of malaria with the fatal cerebral malaria being caused by Plasmodium falciparum (Pf). Despite the availability of antimalarials, emergence of drug resistance has necessitated a constant search for new drugs. All protozoan parasites including Plasmodium sp. are purine auxotophs and are completely dependent on their host for their purine requirements [1]. Hypoxanthine serves as a precursor for the purines, adenosine monophosphate (AMP) and guanosine monophosphate (GMP) in the intra-erythrocytic stages of P. falciparum life cycle. A single phosphoribosyltransferase with specificities for hypoxanthine, guanine and xanthine converts hypoxanthine to inosine monophosphate (IMP) which is the common precursor for both AMP and GMP. IMP is converted to xanthosine monophosphate (XMP) by the action of IMP dehydrogenase followed by GMP synthetase (GMPS) that catalyzes the conversion of XMP to GMP. Precluding GMP formation by inhibiting the enzyme IMP dehydrogenase has been shown to be lethal to P. falciparum establishing PfGMPS as a good drug target [2]. PfGMPS has two functional domains; a glutamine amidotransferase domain (GATase) which hydrolyzes glutamine to glutamate and ammonia, and an ATP Pyrophosphatase (ATPPase) domain which catalyzes the formation of adenyl-XMP intermediate. The ammonia generated by GATase is channelled to ATPPase where it attacks the adenyl-XMP intermediate generating GMP. GMP synthetase being a multi-faceted enzyme involving two active site chemistries, allostery and ammonia channelling is an interesting candidate for studies from a mechanistic perspective. Although the first crystal structure of E. coli GMPS was published twenty years ago and crystal structures of GMPS from other organisms were available, they failed to provide a molecular understanding of the allosteric activation of GATase activity upon ATP, XMP binding to ATPPase domain. In many ammonia channelling enzymes, the path traversed by ammonia has been demarcated in crystal structures of either apo or substrate-complexed forms. However, an ammonia channel is not evident in the available crystal structures of GMP synthetase. We employed a comprehensive approach involving crystallography, mutagenesis and biochemical assays with the overarching goal of answering the above questions. The crystal structure of PfGMPS_C89A mutant complexed with glutamine showed a conformational change not observed earlier wherein the GATase domain was rotated by 85 degrees in comparison with the apo-structure. The structural observations were validated using mutagenesis and biochemical assays. The wild type and mutants were subjected to both steady-state as well as transient kinetics to quantitate the rates of GMP, glutamate and adenyl-XMP formation. These experiments explicitly established the roles of helix 371-375 and loop 376-401 present in the ATPPase domain in catalysis and allostery. Further, these studies have enabled us to hypothesize how rotation of the GATase domain in conjunction with closing of the loop 376-401 in the ATPPase domain forms a closed channel leading to ammonia transfer [3]. The results of these experiments will be presented in the poster.

[1] Gardner, M.J. et al. (2002). Nature. 419, 498-511.

[2] Webster, H.K. \& Whaun, J.M. (1982). J Clin Invest. 70, 461-469.

[3] Ballut, L. et al. (2015). Nat Commun. 6, 8930.

Keywords: GMP synthetase, amidotransferase, ammonia channeling 